\title{
Fate of most massive stars
}

\author{
Norhasliza Yusof $^{1}$, Raphael Hirschi ${ }^{2,3}$ and Hasan Abu Kassim ${ }^{1}$ \\ ${ }^{1}$ Department of Physics, University of Malaya, Kuala Lumpur, Malaysia \\ email: norhasliza@siswa.um.edu.my \\ ${ }^{2}$ Astrophysics Group, EPSAM, Lennard-Jones Laboratories, Keele University \\ ST5 2BG Keele, Staffordshire, UK \\ ${ }^{3}$ Institute for the Physics and Mathematics of the Universe, University of Tokyo, Kashiwa, \\ 277-8568, Japan
}

\begin{abstract}
The first generations of stars are thought to have been more massive than Pop I stars and therefore some of these are thought to have produced pair creation supernovae (PCSNe) at the end of their life. However, the chemical signature of PCSNe is not observed in extremely metal poor stars (e.g. Umeda and Nomoto 2002) and it raises the following questions: Were stars born less (or more massive) than the mass range expected to lead to the PCSNe? Or was mass loss too strong during the evolution of these stars and prevented them from retaining enough mass to produce PCSNe? The discovery of very massive stars (VMS, $M>100 M_{\odot}$ ) in the Milky Way and LMC (Crowther et al. 2010) shows that VMS can form and exist. The observations of PCSN candidates (2006gy \& 2007bi) also seems to indicate that such SNe may occur. Mass loss plays a crucial role in the life of VMS since the star will only die as a PCSN if the star retains a high mass throughout its life. In this paper, we shall describe the dependence of VMS evolution on metallicity and present stellar evolution models at various metallicities, including the effects of mass loss and rotation. Based on our models, we will give our predictions concerning the fate of these VMS, either a PCSN or SNIc (possibly GRBs in some cases) as a function of metallicity.
\end{abstract}

Keywords. stars: evolution, rotation, supernovae

\section{Introduction}

The fate of very massive stars depends on their mass, composition and rotational rate. Stars with an initial mass in the range 10-140 $\mathrm{M}_{\odot}$ produce a central iron core and eventually collapse (Heger \& Woosley 2002). This type of collapse will result in a core collapse supernova (CC SN) of Type II, Ib or Ic or black hole (BH). The classification of these types of supernova depends on the existence of the hydrogen envelope in the star. Stars that undergo the core collapse at the end of their evolution will become either neutron stars or black holes. A star with initial mass in the range of 140-260 $\mathrm{M}_{\odot}$ will become unstable due to the electron-positron pair creation in their oxygen-rich core (Barkat, Rakavy \& Sack 1967). The instability starts when the central temperature increases above $5 \times 10^{9} \mathrm{~K}$ and leads to the explosion of the oxygen core to form a pair creation supernova (PCSN). The analysis of which models will end up as PCSN is done by considering the $\mathrm{M}_{\mathrm{CO}}$ of the final model. Our models include both rotating and nonrotating models with mass range of $120-500 \mathrm{M}_{\odot}$ for SMC, LMC and solar metallicities.

\section{Fate of very massive stars and PCSN candidates}

We computed main sequence evolutionary models in order to determine the mass of the most massive stars known to date, R136a1 (Crowther et al. 2010). In this work (Yusof et al. in prep.), we evolved the models beyond the main sequence until at least the end of He-burning and in most cases until the start of O-burning. All the computed models 


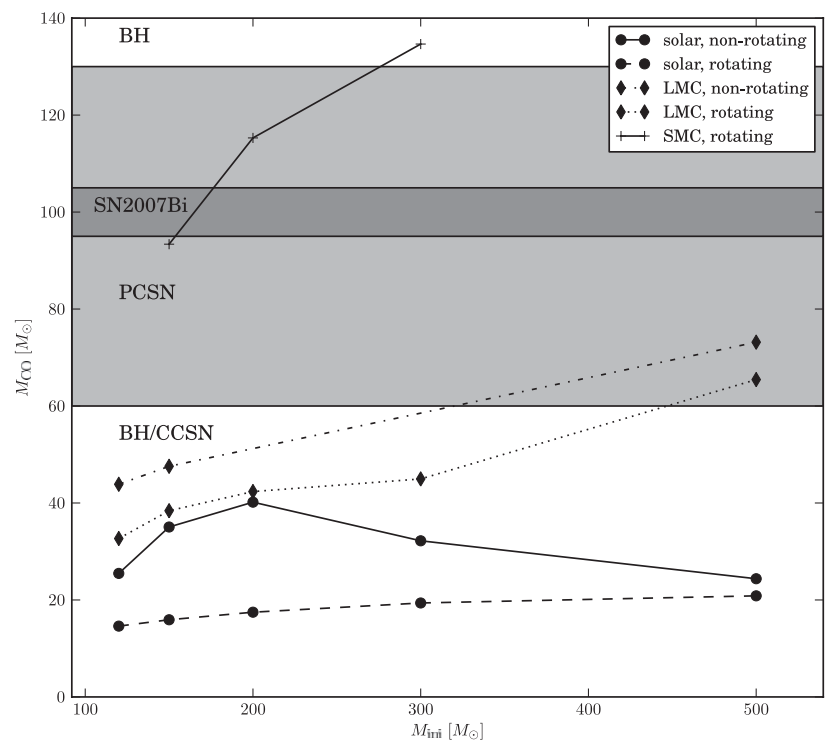

Figure 1. Fate of our models.

lose their H-rich envelope and end up as either as SN Ic, SNIc/Ib or SN Ib. Thus our models contradict the idea that SN2006gy (Smith et al. 2007) is a PCSN. In order to predict the fate of our models (PCSN or CCSN), we consider $\mathrm{M}_{\mathrm{CO}}$ (Yoshida \& Umeda 2011). In Fig. 1 the $\mathrm{M}_{\mathrm{CO}}$ mass range expected to lead to PCSN is indicated. At $Z_{\odot}$, mass loss is too strong and all stars end as BH/CCSN. At $Z_{\mathrm{LMC}}$, only the $500 \mathrm{M}_{\odot}$ models (both rotating and non-rotating) and at $Z_{\mathrm{SMC}}$ the 150 and $200 \mathrm{M}_{\odot}$ rotating models end as PCSN. We also include in Fig. 1 the suggested $\mathrm{M}_{\mathrm{CO}}$ (Yoshida \& Umeda 2011) for SN2007bi (Gal-Yam et al. 2009) since $Z_{\mathrm{SMC}}<Z_{07 \mathrm{bi}}=0.04<Z_{\mathrm{LMC}}$. We can see that possible progenitors for SN2007bi are $160-175 \mathrm{M}_{\odot}$ rotating models at $Z_{\mathrm{SMC}}$.

Our models show that the mass loss and its metallicity dependence play a crucial role in determining the fate of very massive stars.

\section{Acknowledgments}

N.Yusof would like to thank the organizers of IAU279 for granting the full financial support to attend this conference, UM and MOHE for the SLAI fellowship.

\section{References}

Barkat, Z., Rakavy, G., \& Sack, N. 1967, Physical Review Letters, 18, 379

Crowther, P. A., et al. 2010, Mon. Not. R. Astron. Soc, 408, 731

Gal-Yam A., et al. 2009, Nature, 462, 624

Heger, A. \& Woosley, S. E. 2002, ApJ, 567, 532

Smith, N., et al. 2007, ApJ, 666, 1116

Umeda, H. \& Nomoto, K. 2002, ApJ, 565, 385

Yoshida, T. \& Umeda, H. 2011, Mon. Not. R. Astron. Soc, 412, L78 